\title{
Some identities related to Dedekind sums and the second-order linear recurrence polynomials
}

\author{
Jianghua $\mathrm{Li}^{1 *}$ and Han Zhang ${ }^{2}$
}

\section{"Correspondence:}

jianghuali@xaut.edu.cn

'School of Science, Xi'an University

of Technology, Xi'an, Shaanxi,

P.R. China

Full list of author information is

available at the end of the article

\begin{abstract}
In this paper, we use the elementary method and the reciprocity theorem of Dedekind sums to study the computational problem of one kind Dedekind sums, and give two interesting computational formulae related to Dedekind sums and the second-order linear recurrence polynomials.

MSC: Primary 11B37; 11F20
\end{abstract}

Keywords: identity; Dedekind sums; the second-order linear recurrence polynomials

\section{Introduction}

For any positive integer $x$, we define the generalized Lucas polynomial $L_{n}(x)$ as follows: $L_{0}(x)=2, L_{1}(x)=x$, and $L_{n+1}(x)=x L_{n}(x)+L_{n-1}(x)$ for all $n \geq 1$.

It is clear that this polynomial is a second-order linear recurrence polynomial, it is satisfying the computational formula:

$$
L_{n}(x)=\left(\frac{x+\sqrt{x^{2}+4}}{2}\right)^{n}+\left(\frac{x-\sqrt{x^{2}+4}}{2}\right)^{n} .
$$

$L_{n}(1)=L_{n}$ is the well-known Lucas sequence; $L_{n}(2)=Q_{n}$ is the Lucas-Pell sequence. About the properties of this sequence and related contents, some authors had studied them, and obtained many interesting results, see [1-3]. In this paper, we use the elementary method and the reciprocity theorem of Dedekind sums to study the computational problem of one kind Dedekind sums, and obtain some interesting identities related to Dedekind sums and the second-order linear recurrence polynomials. For convenience, we first give the definition of the Dedekind sums $S(h, q)$ as follows:

For a positive integer $q$ and integer $h$ with $(q, h)=1$, the classical Dedekind sum $S(h, q)$ is defined by

$$
S(h, q)=\sum_{a=1}^{q}\left(\left(\frac{a}{q}\right)\right)\left(\left(\frac{a h}{q}\right)\right),
$$

where

$$
((x))= \begin{cases}x-[x]-\frac{1}{2}, & \text { if } x \text { is not an integer } \\ 0, & \text { if } x \text { is an integer. }\end{cases}
$$

O2013 Li and Zhang; licensee Springer. This is an Open Access article distributed under the terms of the Creative Commons Attribution License (http://creativecommons.org/licenses/by/2.0), which permits unrestricted use, distribution, and reproduction in any medium, provided the original work is properly cited. 
This sum describes the behaviour of the logarithm of eta-function (see [4]) under modular transformations. About its other properties and applications, see [5-8]. For example, Carlitz [5] proved the reciprocity theorem

$$
S(h, q)+S(q, h)=\frac{h^{2}+q^{2}+1}{12 h q}-\frac{1}{4}
$$

where $h>0, q>0$ and $(h, q)=1$.

In this paper, we shall give an exact computational formula for $S\left(L_{n}(x), L_{n+1}(x)\right)$. That is, we shall prove the following two theorems.

Theorem 1 For any integers $n \geq 0$ and odd number $x \geq 1$, we have the computational formula

$$
S\left(L_{2 n}(x), L_{2 n+1}(x)\right)=\frac{1}{12} \cdot \frac{x^{2}+5}{x^{2}+4} \cdot \frac{L_{2 n}(x)}{L_{2 n+1}(x)}+\frac{x^{3}-6 x^{2}+5 x-24}{24\left(x^{2}+4\right)} \text {. }
$$

Theorem 2 For any integers $n \geq 0$ and odd number $x \geq 1$, we have the computational formula

$$
S\left(L_{2 n-1}(x), L_{2 n}(x)\right)=\frac{1}{12} \cdot \frac{x^{2}+3}{x^{2}+4} \cdot \frac{L_{2 n-1}(x)}{L_{2 n}(x)}+\frac{x}{24} \cdot \frac{x^{2}+3}{x^{2}+4} .
$$

From the theorems, we may immediately deduce the following two corollaries.

Corollary 1 For any positive integer $n$, we have the identities

$$
S\left(L_{n-1}, L_{n}\right)= \begin{cases}\frac{1}{10} \cdot \frac{L_{2 m}}{L_{2 m+1}}-\frac{1}{5}, & \text { if } n=2 m+1 \\ \frac{1}{15} \cdot \frac{L_{2 m-1}}{L_{2 m}}+\frac{1}{30}, & \text { if } n=2 m\end{cases}
$$

Corollary 2 For any odd number $x \geq 1$, we have the limits

$$
\begin{aligned}
& \lim _{n \rightarrow \infty} S\left(L_{2 n-1}(x), L_{2 n}(x)\right)=\frac{1}{24} \cdot \frac{x^{2}+3}{\sqrt{x^{2}+4}} \text { and } \\
& \lim _{n \rightarrow \infty} S\left(L_{2 n}(x), L_{2 n+1}(x)\right)=\frac{1}{24} \cdot \frac{x^{2}+5-6 \cdot \sqrt{x^{2}+4}}{\sqrt{x^{2}+4}} .
\end{aligned}
$$

In our theorems, $x$ must be a positive odd number. If $x$ is an even number, then $\left(L_{n}(x), L_{n+1}(x)\right)=\cdots=(2, x)=2$. This time, the situation is more complex, it is very difficult for us to give an exact computational formula for $S\left(L_{n}(x), L_{n+1}(x)\right)$.

\section{Proof of the theorems}

In this section, we shall prove our theorems directly. First, we prove Theorem 1. It is clear that for any positive integer $n$ and odd number $x$, we have $\left(L_{n}(x), L_{n+1}(x)\right)=$ $\left(L_{n-1}(x), L_{n}(x)\right)=\cdots=\left(L_{0}(x), L_{1}(x)\right)=(2, x)=1$. So, by reciprocity theorem $(1)$, we have

$$
\begin{aligned}
& S\left(L_{2 n}(x), L_{2 n+1}(x)\right)+S\left(L_{2 n+1}(x), L_{2 n}(x)\right) \\
& \quad=\frac{1}{12}\left[\frac{L_{2 n+1}(x)}{L_{2 n}(x)}+\frac{L_{2 n}(x)}{L_{2 n+1}(x)}+\frac{1}{L_{2 n+1}(x) L_{2 n}(x)}\right]-\frac{1}{4}
\end{aligned}
$$




$$
\begin{aligned}
& =\frac{1}{12}\left[\frac{x L_{2 n}(x)+L_{2 n-1}(x)}{L_{2 n}(x)}+\frac{L_{2 n}(x)}{L_{2 n+1}(x)}+\frac{1}{L_{2 n+1}(x) L_{2 n}(x)}\right]-\frac{1}{4} \\
& =\frac{1}{12}\left[\frac{L_{2 n}(x)}{L_{2 n+1}(x)}+\frac{L_{2 n-1}(x)}{L_{2 n}(x)}+\frac{1}{L_{2 n+1}(x) L_{2 n}(x)}\right]+\frac{x}{12}-\frac{1}{4} .
\end{aligned}
$$

Similarly, we also have the identity

$$
\begin{aligned}
& S\left(L_{2 n-1}(x), L_{2 n}(x)\right)+S\left(L_{2 n}(x), L_{2 n-1}(x)\right) \\
& \quad=\frac{1}{12}\left[\frac{L_{2 n-1}(x)}{L_{2 n}(x)}+\frac{L_{2 n-2}(x)}{L_{2 n-1}(x)}+\frac{1}{L_{2 n}(x) L_{2 n-1}(x)}\right]+\frac{x}{12}-\frac{1}{4} .
\end{aligned}
$$

Note that $S\left(L_{2 n}(x), L_{2 n-1}(x)\right)=S\left(L_{2 n-2}(x), L_{2 n-1}(x)\right)$ and $S\left(L_{2 n+1}(x), L_{2 n}(x)\right)=S\left(L_{2 n-1}(x)\right.$, $L_{2 n}(x)$ ), from identities (2) and (3), we have

$$
\begin{aligned}
S & \left(L_{2 n}(x), L_{2 n+1}(x)\right)-S\left(L_{2 n-2}(x), L_{2 n-1}(x)\right) \\
& =\frac{1}{12}\left[\frac{L_{2 n}(x)}{L_{2 n+1}(x)}-\frac{L_{2 n-2}(x)}{L_{2 n-1}(x)}+\frac{1}{L_{2 n+1}(x) L_{2 n}(x)}-\frac{1}{L_{2 n}(x) L_{2 n-1}(x)}\right] \\
& =\frac{1}{12}\left[\frac{L_{2 n}(x)}{L_{2 n+1}(x)}-\frac{L_{2 n-2}(x)}{L_{2 n-1}(x)}-\frac{x}{L_{2 n+1}(x) L_{2 n-1}(x)}\right] \\
& =\frac{1}{12}\left[\frac{L_{2 n}(x)}{L_{2 n+1}(x)}-\frac{L_{2 n-2}(x)}{L_{2 n-1}(x)}+\frac{x}{x^{3}+4 x}\left(\frac{L_{2 n}(x)}{L_{2 n+1}(x)}-\frac{L_{2 n-2}(x)}{L_{2 n-1}(x)}\right)\right] \\
& =\frac{1}{12} \cdot \frac{x^{2}+5}{x^{2}+4} \cdot\left[\frac{L_{2 n}(x)}{L_{2 n+1}(x)}-\frac{L_{2 n-2}(x)}{L_{2 n-1}(x)}\right] .
\end{aligned}
$$

From (4), we may immediately deduce the recurrence formula

$$
\begin{aligned}
& S\left(L_{2 n}(x), L_{2 n+1}(x)\right)-\frac{1}{12} \cdot \frac{x^{2}+5}{x^{2}+4} \cdot \frac{L_{2 n}(x)}{L_{2 n+1}(x)} \\
& \quad=S\left(L_{2 n-2}(x), L_{2 n-1}(x)\right)-\frac{1}{12} \cdot \frac{x^{2}+5}{x^{2}+4} \cdot \frac{L_{2 n-2}(x)}{L_{2 n-1}(x)} .
\end{aligned}
$$

Using (5), repeatedly, and note that formula (1) and $S(x, 2)=0$, we have

$$
\begin{aligned}
& S\left(L_{2 n}(x), L_{2 n+1}(x)\right)-\frac{1}{12} \cdot \frac{x^{2}+5}{x^{2}+4} \cdot \frac{L_{2 n}(x)}{L_{2 n+1}(x)} \\
& \quad=\cdots \\
& \quad=S\left(L_{0}(x), L_{1}(x)\right)-\frac{1}{12} \cdot \frac{x^{2}+5}{x^{2}+4} \cdot \frac{L_{0}(x)}{L_{1}(x)}=S(2, x)-\frac{1}{12} \cdot \frac{x^{2}+5}{x^{2}+4} \cdot \frac{2}{x} \\
& \quad=\frac{x^{2}+5}{24 x}-\frac{1}{4}-\frac{1}{12} \cdot \frac{x^{2}+5}{x^{2}+4} \cdot \frac{2}{x}=\frac{x^{3}-6 x^{2}+5 x-24}{24\left(x^{2}+4\right)}
\end{aligned}
$$

or

$$
S\left(L_{2 n}(x), L_{2 n+1}(x)\right)=\frac{1}{12} \cdot \frac{x^{2}+5}{x^{2}+4} \cdot \frac{L_{2 n}(x)}{L_{2 n+1}(x)}+\frac{x^{3}-6 x^{2}+5 x-24}{24\left(x^{2}+4\right)} .
$$

This proves Theorem 1. 
Now, we prove Theorem 2. From the method of proving (2), we have

$$
\begin{aligned}
& S\left(L_{2 n-1}(x), L_{2 n}(x)\right)+S\left(L_{2 n}(x), L_{2 n-1}(x)\right) \\
& \quad=\frac{1}{12}\left[\frac{L_{2 n}(x)}{L_{2 n-1}(x)}+\frac{L_{2 n-1}(x)}{L_{2 n}(x)}+\frac{1}{L_{2 n}(x) L_{2 n-1}(x)}\right]-\frac{1}{4} \\
& =\frac{1}{12}\left[\frac{x L_{2 n-1}(x)+L_{2 n-2}(x)}{L_{2 n-1}(x)}+\frac{L_{2 n-1}(x)}{L_{2 n}(x)}+\frac{1}{L_{2 n}(x) L_{2 n-1}(x)}\right]-\frac{1}{4} \\
& =\frac{1}{12}\left[\frac{L_{2 n-1}(x)}{L_{2 n}(x)}+\frac{L_{2 n-2}(x)}{L_{2 n-1}(x)}+\frac{1}{L_{2 n}(x) L_{2 n-1}(x)}\right]+\frac{x}{12}-\frac{1}{4}
\end{aligned}
$$

and

$$
\begin{aligned}
S & \left(L_{2 n-2}(x), L_{2 n-1}(x)\right)+S\left(L_{2 n-1}(x), L_{2 n-2}(x)\right) \\
& =\frac{1}{12}\left[\frac{L_{2 n-1}(x)}{L_{2 n-2}(x)}+\frac{L_{2 n-2}(x)}{L_{2 n-1}(x)}+\frac{1}{L_{2 n-1}(x) L_{2 n-2}(x)}\right]-\frac{1}{4} \\
& =\frac{1}{12}\left[\frac{x L_{2 n-2}(x)+L_{2 n-3}(x)}{L_{2 n-2}(x)}+\frac{L_{2 n-2}(x)}{L_{2 n-1}(x)}+\frac{1}{L_{2 n-1}(x) L_{2 n-2}(x)}\right]-\frac{1}{4} \\
& =\frac{1}{12}\left[\frac{L_{2 n-2}(x)}{L_{2 n-1}(x)}+\frac{L_{2 n-3}(x)}{L_{2 n-2}(x)}+\frac{1}{L_{2 n-1}(x) L_{2 n-2}(x)}\right]+\frac{x}{12}-\frac{1}{4} .
\end{aligned}
$$

Note that $S\left(L_{2 n}(x), L_{2 n-1}(x)\right)=S\left(L_{2 n-2}(x), L_{2 n-1}(x)\right), S\left(x^{2}+2, x\right)=S(2, x), S(x, 2)=0$, from (1), (6) and (7), we have

$$
\begin{aligned}
S & \left(L_{2 n-1}(x), L_{2 n}(x)\right)-S\left(L_{2 n-1}(x), L_{2 n-2}(x)\right) \\
& =\frac{1}{12}\left[\frac{L_{2 n-1}(x)}{L_{2 n}(x)}-\frac{L_{2 n-3}(x)}{L_{2 n-2}(x)}+\frac{1}{L_{2 n}(x) L_{2 n-1}(x)}-\frac{1}{L_{2 n-1}(x) L_{2 n-2}(x)}\right] \\
& =\frac{1}{12}\left[\frac{L_{2 n-1}(x)}{L_{2 n}(x)}-\frac{L_{2 n-3}(x)}{L_{2 n-2}(x)}-\frac{x}{L_{2 n}(x) L_{2 n-2}(x)}\right] \\
& =\frac{1}{12}\left[\frac{L_{2 n-1}(x)}{L_{2 n}(x)}-\frac{L_{2 n-3}(x)}{L_{2 n-2}(x)}-\frac{1}{x^{2}+4}\left(\frac{L_{2 n-1}(x)}{L_{2 n}(x)}-\frac{L_{2 n-3}(x)}{L_{2 n-2}(x)}\right)\right] \\
& =\frac{1}{12} \cdot \frac{x^{2}+3}{x^{2}+4} \cdot\left(\frac{L_{2 n-1}(x)}{L_{2 n}(x)}-\frac{L_{2 n-3}(x)}{L_{2 n-2}(x)}\right)
\end{aligned}
$$

or

$$
\begin{aligned}
& S\left(L_{2 n-1}(x), L_{2 n}(x)\right)-\frac{1}{12} \cdot \frac{x^{2}+3}{x^{2}+4} \cdot \frac{L_{2 n-1}(x)}{L_{2 n}(x)} \\
& \quad=S\left(L_{2 n-3}(x), L_{2 n-2}(x)\right)-\frac{1}{12} \cdot \frac{x^{2}+3}{x^{2}+4} \cdot \frac{L_{2 n-3}(x)}{L_{2 n-2}(x)} \\
& \quad=\ldots \\
& \quad=S\left(x, x^{2}+2\right)-\frac{1}{12} \cdot \frac{x^{2}+3}{x^{2}+4} \cdot \frac{x}{x^{2}+2} \\
& \quad=S\left(x, x^{2}+2\right)+S\left(x^{2}+2, x\right)-S(2, x)-S(x, 2)-\frac{1}{12} \cdot \frac{x^{2}+3}{x^{2}+4} \cdot \frac{x}{x^{2}+2} \\
& \quad=\frac{x^{2}+\left(x^{2}+2\right)^{2}+1}{12 x\left(x^{2}+2\right)}-\frac{x^{2}+5}{24 x}-\frac{1}{12} \cdot \frac{x^{2}+3}{x^{2}+4} \cdot \frac{x}{x^{2}+2}=\frac{x}{24} \cdot \frac{x^{2}+3}{x^{2}+4}
\end{aligned}
$$


or

$$
S\left(L_{2 n-1}(x), L_{2 n}(x)\right)=\frac{1}{12} \cdot \frac{x^{2}+3}{x^{2}+4} \cdot \frac{L_{2 n-1}(x)}{L_{2 n}(x)}+\frac{x}{24} \cdot \frac{x^{2}+3}{x^{2}+4} .
$$

This completes the proof of Theorem 2 .

Note that the definition of $L_{n}(x), x>1$, and the limit

$$
\lim _{n \rightarrow+\infty} \frac{L_{2 n}(x)}{L_{2 n+1}(x)}=\lim _{n \rightarrow+\infty} \frac{L_{2 n-1}(x)}{L_{2 n}(x)}=\frac{2}{x+\sqrt{x^{2}+4}}=\frac{-x+\sqrt{x^{2}+4}}{2},
$$

from our theorems, we may immediately deduce Corollary 2.

\section{Competing interests}

The authors declare that they have no competing interests.

\section{Authors' contributions}

$J \mathrm{~L}$ carried out the exact computational formula for $S\left(L_{n}(x), L_{n+1}(x)\right), S\left(L_{2 n-1}(x), L_{2 n}(x)\right)$. HZ participated in the research and summary of the study. All authors read and approved the final manuscript.

\section{Author details}

${ }^{1}$ School of Science, Xi'an University of Technology, Xi'an, Shaanxi, P.R. China. ${ }^{2}$ Department of Mathematics, Northwest University, Xi'an, Shaanxi, P.R. China.

\section{Acknowledgements}

The authors would like to thank the referee for his very helpful and detailed comments, which have significantly improved the presentation of this paper. This work is supported by the P.S.F. (2013JZ001, 2013KJXX-34) and the N.S.F. (11071194) of P.R. China.

Received: 7 August 2013 Accepted: 9 September 2013 Published: 08 Nov 2013

\section{References}

1. Yi, Y, Zhang, W: Some identities involving the Fibonacci polynomials. Fibonacci Q. 40, 314-318 (2002)

2. $M a, R$, Zhang, W: Several identities involving the Fibonacci numbers and Lucas numbers. Fibonacci Q. 45, 164-170 (2007)

3. Ohtsuka, H, Nakamura, S: On the sum of reciprocal Fibonacci numbers. Fibonacci Q. 46/47, 153-159 (2008/2009)

4. Rademacher, H: On the transformation of $\log \eta(\tau)$. J. Indian Math. Soc. 19, 25-30 (1955)

5. Carlitz, L: The reciprocity theorem of Dedekind sums. Pac. J. Math. 3, 513-522 (1953)

6. Jia, C: On the mean value of Dedekind sums. J. Number Theory 87, 173-188 (2001)

7. Zhang, W: A note on the mean square value of the Dedekind sums. Acta Math. Hung. 86, 275-289 (2000)

8. Zhang, W: On the mean values of Dedekind sums. J. Théor. Nr. Bordx. 8, 429-442 (1996)

10.1186/1687-1847-2013-299

Cite this article as: Li and Zhang: Some identities related to Dedekind sums and the second-order linear recurrence polynomials. Advances in Difference Equations 2013, 2013:299

\section{Submit your manuscript to a SpringerOpen ${ }^{\circ}$ journal and benefit from:}

- Convenient online submission

Rigorous peer review

- Immediate publication on acceptance

- Open access: articles freely available online

- High visibility within the field

- Retaining the copyright to your article 